\title{
Transcriptome and de novo analysis of Rosa xanthina f. spontanea in response to cold stress
}

\author{
Defeng Zhuang ${ }^{1,2,3}$, Ce Ma ${ }^{1}$, Li Xue ${ }^{3}$, Zhen Li ${ }^{1}$, Cheng Wang ${ }^{4}$, Jiajun Lei ${ }^{3^{*}}$ and Xingfu Yuan ${ }^{1 *}$
}

\begin{abstract}
Background: Rose is one of most popular ornamental plants worldwide and is of high economic value and great cultural importance. However, cold damage restricts its planting application in cold areas. To elucidate the metabolic response of rose under low temperature stress, we conducted transcriptome and de novo analysis of Rosa xanthina f. spontanea.

Results: A total of 124,106 unigenes from 9 libraries were generated by de novo assembly, with N50 length was $1470 \mathrm{bp}$, under $4^{\circ} \mathrm{C}$ and $-20^{\circ} \mathrm{C}$ stress $\left(23^{\circ} \mathrm{C}\right.$ was used as a control). Functional annotation and prediction analyses identified 55,084 unigenes, and $67.72 \%$ of these unigenes had significant similarity (BLAST, $E \leq 10^{-5}$ ) to those in the public databases. A total of 3031 genes were upregulated and 3891 were downregulated at $4^{\circ} \mathrm{C}$ compared with $23^{\circ} \mathrm{C}$, and 867 genes were upregulated and 1763 were downregulated at $-20^{\circ} \mathrm{C}$ compared with $23^{\circ} \mathrm{C}$. A total of 468 common DEGs were detected under cold stress, and the matched DEGs were involved in three functional categories: biological process (58.45\%), cellular component (11.27\%) and molecular function (30.28\%). Based on KEGG functional annotations, four pathways were significantly enriched: metabolic pathway, response to plant pathogen interaction (32 genes); starch and sucrose metabolism (21 genes); circadian rhythm plant (8 genes); and photosynthesis antenna proteins (7 genes).

Conclusions: Our study is the first to report the response to cold stress at the transcriptome level in $R$. xanthina f. spontanea. The results can help to elucidate the molecular mechanism of cold resistance in rose and provide new insights and candidate genes for genetically enhancing cold stress tolerance.
\end{abstract}

Keywords: Rose, Low-temperature stress, DEGs, Metabolic pathway

\section{Background}

Rose is one of the most common ornamental plants, has high economic value, and is very popular with people worldwide. However, due to a lack of cold tolerance, its planting application is affected by low temperatures in cold regions. Cold resistance in woody plants is a complex metabolic process. Generally, plant growth and development stop when winter arrives, and cold tolerance

\footnotetext{
*Correspondence: jiajunleisy@163.com; laasy@189.cn

1 Liaoning Academy of Agricultural Sciences, Shenyang 110161, Liaoning, China

${ }^{3}$ College of Horticulture, Shenyang Agricultural University,

Shenyang 110866, Liaoning, China

Full list of author information is available at the end of the article
}

and dormancy gradually form [1]. In addition, this process will result in variation in morphological traits at the transcriptional/biochemical level that then improve the stability of the membrane system, which can survive safely in winter, such as in most temperate woody plants [2].

A transcriptome represents the sum of all RNA that is transcribed in a functional state in a specific tissue or cell at a certain stage; it mainly includes mRNA and noncoding RNA [3]. Expression of the whole genome was revealed at the whole transcriptional level under abiotic stress to identify transcripts related to cold tolerance. It is of great significance to construct the transcriptional regulatory network of the genome under abiotic stress genome in terms of the complex regulatory network 
involved in increasing abiotic stress adaptation and tolerance $[4,5]$. Transcriptome sequencing has already been performed for plants under low-temperature stress, such as in Camellia sinensis and Populus euphratica [6, 7].

Recently, there have been many studies on the transcriptome in rose. Abundant genetic information has been obtained on resistance and development with respect to transcriptome sequencing by using roots, leaves, flowers and fruits of rose as materials [8-11]. The fusion strategy combining the genome and proteome can provide a certain theoretical basis for resolving the biological problems of rose in the future. Transcriptome sequencing analysis has been used to study cold stress in the leaves in $R$. multiflora [12], fruit in blueberry [13] and floral buds in Rosa hybrida [14]. To date, transcriptomic information in rose has not been clarified because of the complexity of cold resistance mechanisms.

Rosa xanthina, a wild species of Sect. Pimpinellifoliae, is native to northeastern and northern China. In addition, $R$. xanthina $\mathrm{f}$. spontanea has high cold/drought tolerance and disease resistance and is an important germplasm resource in the breeding of modern rose [15]. In the present study, we carried out transcriptome sequencing analysis of $R$. xanthina $\mathrm{f}$. spontanea under low-temperature stress to clarify the functions and metabolic pathways associated with DEGs, which can provide a theoretical foundation for the cold-resistance mechanism in rose.

\section{Results}

\section{Transcriptome sequencing and assembly}

The original data obtained by sequencing with an Illumina HiSeq 4000 were transformed into raw reads by base calling. The total number of nucleotides obtained from nine libraries was $64.52 \mathrm{G}$, and the total number of nucleotides was between $5.23 \mathrm{G}$ and $7.59 \mathrm{G}$ in each sample. The Q30 ratio of each sample was greater than 94\%, and the GC content was relatively consistent, at approximately $47 \%$ (Table S1). A total of 124,106 transcripts and 55,084 nonredundant unigenes were obtained from nine libraries. The average length of the unigenes was $661 \mathrm{bp}$, and the N50 length was $1470 \mathrm{bp}$. Consequently, the sequencing data quality was high and met the requirements for subsequent analysis. There were 29,582 unigenes with lengths of $200-500 \mathrm{bp}$, accounting for $53.70 \%$; 10,103 unigenes with lengths of $500-1000 \mathrm{bp}$, accounting for $18.34 \%$; 10,112 unigenes ranging from 1000 to $2000 \mathrm{bp}$, accounting for 18.35\%; and 5287 unigenes with lengths greater than $2000 \mathrm{bp}$, accounting for $9.6 \%$ (Table S2).

\section{Functional annotation of unigenes}

The Nr, Swiss-Prot (a manually annotated and reviewed protein sequence database), Pfam (protein family), KOG
(Clusters of Orthologous Groups of proteins), KEGG and GO databases were used to annotate all unigenes with comprehensive gene function information. In the present study, a total of 37,303 unigenes were successfully annotated in the $R$. xanthina $\mathrm{f}$. spontanea database, representing $67.72 \%$ of all unigenes $(55,084)$. Furthermore, Fragaria_vesca presented the highest frequency in the annotation results, with a total of 19,964 comments, accounting for $53.52 \%$ of all the sequences, followed by Nelumbo nucifera (9.0\%), Prunus persica (3.27\%), Phaseolus vulgaris (3.13\%), Vitis vinifera (2.78\%) and Prunus mume (2.36\%) (Fig. S1; Table S3).

A total of 31,258 (56.75\%) unigenes were assigned to GO terms in the cellular component, molecular function and biological process categories; these unigenes were further classified into $50 \mathrm{GO}$ terms (Fig. S2).

Within the cellular component category, a total of 22,417 DEGs were assigned under $4^{\circ} \mathrm{C}$ and $-20^{\circ} \mathrm{C}$ stress $\left(23^{\circ} \mathrm{C}\right.$ as the control), which indicated that the union of all the DEGs was mainly related to the nucleus, cytoplasm, integral component of membrane and chloroplast. For the molecular function, most of the DEGs were enriched for molecular function, protein binding and ATP binding. In the biological process category, biological process, regulation of transcription and DNA-template were the most enriched (Table S4).

To identify the metabolic pathways involved in cold stress of $R$. xanthina f. spontanea leaves, 21,992 DEGs were mapped to the KEGG database, and the mainly 19 different KEGG pathways are assigned in Fig. S3 (Table S5). Among these pathways, carbohydrate metabolism $(2596,11.80 \%)$, translation $(2325,10.57 \%)$ and folding, sorting and degradation (2036, 9.26\%) were the most extensively overrepresented pathways. In addition, metabolic pathways related to environmental adaptation, transport and catabolism were also found. In summary, amino acid metabolism, translation and signal transduction are involved in almost every aspect of plant life. Furthermore, it was demonstrated that highly informative and wide coverage was obtained from transcriptome sequencing of leaves in $R$. xanthina f. spontanea and can be used to analyse the gene products of metabolic pathways and information processing pathways at the molecular level.

\section{DEGs in response to low-temperature stress}

In this study, differentially expressed genes were used for comparative analyses of tolerance to low temperatures $\left(4^{\circ} \mathrm{C}(\mathrm{T} 2),-20^{\circ} \mathrm{C}(\mathrm{T} 3), 23^{\circ} \mathrm{C}\right.$ (T1, control), Fig. 1$)$. A total of 6922 genes were identified at $4^{\circ} \mathrm{C}$; 3031 genes were upregulated, and 3891 were downregulated. In the case of $-20^{\circ} \mathrm{C}, 2630 \mathrm{DEGs}$ were detected, including 867 upregulated genes and 1763 downregulated genes. By comparing the $-20^{\circ} \mathrm{C}$ and $4{ }^{\circ} \mathrm{C}$ treatments, 5741 DEGs were 
Differentially expressed genes in different groups

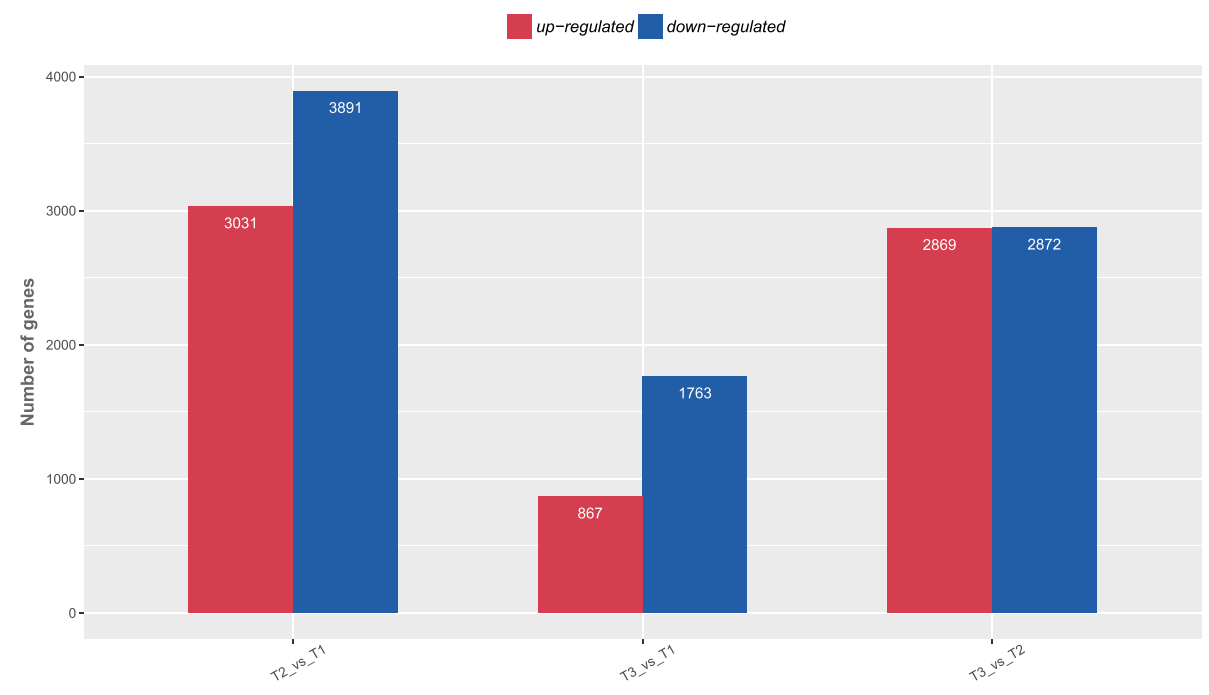

\section{Differential Expressed Genes}

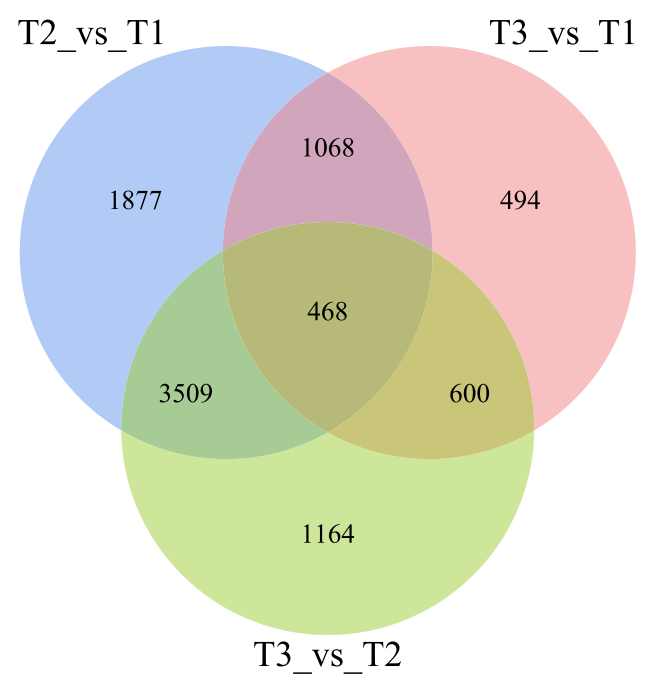

Fig. 1 The column and Venn diagrams of DEGs assembled under low-temperature stress across three sets of comparisons expressed as $4^{\circ} \mathrm{C}$ vs $23^{\circ} \mathrm{C}$ (control), $-20^{\circ} \mathrm{C}$ vs $23^{\circ} \mathrm{C}$ and $4^{\circ} \mathrm{C}$ vs $-20^{\circ} \mathrm{C}$, respectively $(P<0.05)$

identified; 2869 genes were upregulated and 2872 genes were downregulated. All DEGs were less abundant at $-20^{\circ} \mathrm{C}$ than at $4^{\circ} \mathrm{C}$ regardless of whether they were upregulated or downregulated. According to the Venn diagram, 468 DEGs were commonly involved in the above three groups, suggesting that these genes may play an important role in cold tolerance in $R$. xanthina f. spontanea.

\section{Gene ontology enrichment analyses of DEGs}

We elucidated the GO terms related to the biological functions of the DEGs that were significantly altered under the three treatments $\left(23^{\circ} \mathrm{C}, 4^{\circ} \mathrm{C}\right.$ and $\left.-20^{\circ} \mathrm{C}\right)$. The significantly enriched $\mathrm{GO}$ terms $(p<0.05)$ were identified, and classification of the GO terms was performed according to the NCBI nonredundant (NR) annotation using Blast2 GO software. The DEGs were defined as enriched for GO terms when the above conditions were met. A total of 11,705 unigenes were successfully assigned to at least one GO term. All the GO terms were classified into functional groups, including three main categories: biological processes, cellular components and molecular functions. In total, 206 significantly enriched 
GO terms, including 6853 unigenes, at $4{ }^{\circ} \mathrm{C}$ were identified. In the biological process category, there were 69 GO terms (2001). For cellular components, there were $22 \mathrm{GO}$ terms (1734). Within the molecular function category, there were $115 \mathrm{GO}$ terms (3118). The top three most enriched GO terms were response to chitin (52), chloroplast (782), and transcription factor activity and sequence-specific DNA binding (291). Moreover, the results showed that the most enriched GO term was biological process (789) (Table S6).

In comparison, 219 significantly enriched GO terms, including 4708 unigenes, were found at $-20^{\circ} \mathrm{C}$. In the biological process category, there were $128 \mathrm{GO}$ terms (2073). For cellular components, there were 19 GO terms (1150). Within the molecular function category, there were $72 \mathrm{GO}$ terms (1485). The top three most enriched GO terms were transcription factor activity and sequence-specific DNA binding, response to chitin, and defence response to fungus. Moreover, the results showed that the most enriched GO term was plasma membrane (384 genes), and the second was integral component of membrane (327 genes) (Table S7), suggesting that the genes in these processes may play important roles in low temperature perception.

\section{KEGG enrichment analysis of DEGs}

The significantly enriched KEGG metabolic pathways associated with the DEGs in $R$. xanthina $\mathrm{f}$. spontanea were analysed $(p<0.05)$. Under $4{ }^{\circ} \mathrm{C}$ treatment, 1014 DEGs were assigned sixteen pathways (Table 1); 321 genes were upregulated and 693 were downregulated. For the $-20^{\circ} \mathrm{C}$ treatment, 647 DEGs were enriched in 20 pathways; 218 genes were upregulated and 429 genes were downregulated. A total of nine common metabolic pathways were annotated, such as starch and sucrose metabolism and plant-pathogen interactions, and these pathways likely play important roles in low-temperature perception during cold stress treatment. Additionally, the DEGs related to photosynthesis-antenna proteins and circadian rhythm-plant pathways showed that there were more upregulated genes than downregulated genes, and the other pathways showed more downregulated genes than upregulated genes. Under $4^{\circ} \mathrm{C}$ treatment, seven pathways (phenylpropanoid biosynthesis, ribosome biogenesis in eukaryotes, etc.) showed more downregulated genes than upregulated genes. In comparison, the eleven most significantly enriched pathways (plant hormone signal transduction, amino sugar and nucleotide sugar metabolism, etc.) are listed in Table 1 for $-20^{\circ} \mathrm{C}$, and more upregulated genes than downregulated genes were involved in the mapped pathways for monoterpenoid biosynthesis and tryptophan metabolism (Table S8, S9).

\section{GO enrichment analysis of 468 DEGs}

In this study, to reveal which biological functions were significantly related to the common DEGs we obtained, a GO functional enrichment analysis was carried out $(p<0.05)$. The results indicated that the DEGs involved in biological processes, cellular components and molecular functions accounted for $58.45,11.27$, and $30.28 \%$ of the total DEGs, respectively. Consequently, most DEGs were significantly correlated with some biological functions. We found that the DEGs were classified into 93 biological processes, mainly focused on transcriptional regulation, DNA template; transcription, DNA template; response to chitin; response to abscisic acid; response to cold; etc. Regarding cellular components (16), the DEGs were involved in chloroplasts, extracellular regions, chloroplast thylakoid membranes, etc. With respect to molecular functions (43), the DEGs were mainly involved in molecular function, transcription factor activity, sequence-specific DNA binding, and sequence-specific DNA binding (Fig. 2; Table S10).

\section{KEGG pathway enrichment analysis of $468 \mathrm{DEGs}$}

To more precisely investigate the variation in metabolic pathways in leaves during low-temperature stress, statistical pathway enrichment analysis on the DEGs was carried out using the KEGG database. A total of 293 DEGs under low-temperature stress were assigned to 85 different KEGG pathways. There were four significantly enriched pathways $(p<0.05)$ : plant-pathogen interaction, starch and sucrose metabolism, plant circadian rhythm and photosynthesis-antenna proteins (Table 2; Table S11). When the temperature reached $4{ }^{\circ} \mathrm{C}$ and $-20^{\circ} \mathrm{C}$, most DEGs in the plant-pathogen interaction and starch and sucrose metabolism pathways were downregulated. In addition, the genes in the plant circadian rhythm pathway showed similar numbers of, downregulated and upregulated genes. However, seven DEGs in the photosynthesis-antenna protein pathway were upregulated.

\section{Plant-pathogen interaction metabolic pathway}

In the metabolic pathway of plant-pathogen interactions, as shown in Table 2, after removing the genes with TPM values less than 10, we observed 6 upregulated genes and 22 downregulated genes at $4{ }^{\circ} \mathrm{C}$ and 4 upregulated genes and 24 downregulated genes at $-20^{\circ} \mathrm{C}$. The genes cyclic nucleotide-gated ion channel 1 (CNGC1, (TRINITY_DN27111_c1_g1, TRINITY $\left.\left.D N 28981 \_c 1 \_g 5\right)\right)$, leucine-rich repeat receptor-like serine/threonine-protein kinase $(L R R-R L K)$ and inactive receptor kinase $(A t 4 g 23740)$ were significantly upregulated at $4{ }^{\circ} \mathrm{C}$ and $-20^{\circ} \mathrm{C}$, but the genes cyclic nucleotide-gated ion channel 1 (CNGC1, (TRIN$I T Y \_D N$ 32274_c2_g1)) and ethylene-responsive 
Table 1 Summary of KEGG pathway functional annotations under cold stress treatment

\begin{tabular}{|c|c|c|c|c|c|c|c|c|c|}
\hline \multirow[t]{2}{*}{ Pathway } & \multirow[t]{2}{*}{ ID code } & \multicolumn{4}{|c|}{$4^{\circ} \mathrm{C}$ vs. $23^{\circ} \mathrm{C}$} & \multicolumn{4}{|c|}{$-20^{\circ} \mathrm{C}$ vs. $23^{\circ} \mathrm{C}$} \\
\hline & & $\begin{array}{l}\text { Total } \\
\text { number of } \\
\text { DEGs }\end{array}$ & Up & Down & $P$ Value & $\begin{array}{l}\text { Total } \\
\text { number of } \\
\text { DEGs }\end{array}$ & Up & Down & $P$ Value \\
\hline Starch and sucrose metabolism & ko00500 & 221 & 37 & 184 & $1.36 \mathrm{E}-08$ & 75 & 22 & 53 & 0.036091 \\
\hline Photosynthesis - antenna proteins & ko00196 & 21 & 18 & 3 & 0.005443 & 12 & 11 & 1 & 0.002156 \\
\hline Circadian rhythm - plant & ko04712 & 48 & 31 & 17 & 0.016043 & 37 & 22 & 15 & $3.92 \mathrm{E}-08$ \\
\hline Plant-pathogen interaction & ko04626 & 274 & 103 & 171 & 0.017747 & 176 & 42 & 134 & $6.66 \mathrm{E}-16$ \\
\hline Glycerolipid metabolism & ko00561 & 57 & 22 & 35 & 0.025967 & 29 & 12 & 17 & 0.004703 \\
\hline Nitrogen metabolism & ko00910 & 26 & 7 & 19 & 0.027501 & 15 & 0 & 15 & 0.003943 \\
\hline Anthocyanin biosynthesis & ko00942 & 12 & 4 & 8 & 0.040398 & 9 & 3 & 6 & 0.001390 \\
\hline Flavonoid biosynthesis & ko00941 & 28 & 5 & 23 & 0.045727 & 20 & 7 & 13 & 0.000119 \\
\hline Zeatin biosynthesis & ko00908 & 20 & 4 & 16 & 0.045810 & 14 & 8 & 6 & 0.000760 \\
\hline Fatty acid elongation & ko00062 & 28 & 4 & 24 & 0.002586 & - & - & - & - \\
\hline Carotenoid biosynthesis & ko00906 & 32 & 13 & 19 & 0.003827 & - & - & - & - \\
\hline Phenylpropanoid biosynthesis & ko00940 & 84 & 28 & 56 & 0.004435 & - & - & - & - \\
\hline Base excision repair & ko03410 & 30 & 6 & 24 & 0.006088 & - & - & - & - \\
\hline Ribosome biogenesis in eukaryotes & ko03008 & 75 & 25 & 50 & 0.014468 & - & - & - & - \\
\hline Ascorbate and aldarate metabolism & ko00053 & 49 & 13 & 36 & 0.025578 & - & - & - & - \\
\hline Riboflavin metabolism & ko00740 & 9 & 1 & 8 & 0.028072 & - & - & - & - \\
\hline Plant hormone signal transduction & ko04075 & - & - & - & - & 102 & 38 & 64 & 4.96E-07 \\
\hline Amino sugar and nucleotide sugar metabolism & Ko00520 & - & - & - & - & 60 & 14 & 46 & 0.000918 \\
\hline Cutin, suberine and wax biosynthesis & Ko00073 & - & - & - & - & 14 & 5 & 9 & 0.001240 \\
\hline Caffeine metabolism & ko00232 & - & - & - & - & 6 & 3 & 3 & 0.010312 \\
\hline Stilbenoid, diarylheptanoid and gingerol biosynthesis & ko00945 & - & - & - & - & 13 & 5 & 8 & 0.015090 \\
\hline Monoterpenoid biosynthesis & ko00902 & - & - & - & - & 7 & 5 & 2 & 0.018729 \\
\hline Tryptophan metabolism & ko00380 & - & - & - & - & 17 & 11 & 6 & 0.029553 \\
\hline Isoquinoline alkaloid biosynthesis & ko00950 & - & - & - & - & 13 & 3 & 10 & 0.036862 \\
\hline Tropane, piperidine and pyridine alkaloid biosynthesis & ko00960 & - & - & - & - & 11 & 4 & 7 & 0.038950 \\
\hline Brassinosteroid biosynthesis & ko00905 & - & - & - & - & 9 & 2 & 7 & 0.039466 \\
\hline Sesquiterpenoid and triterpenoid biosynthesis & ko00909 & - & - & - & - & 8 & 1 & 7 & 0.049619 \\
\hline Total & & 1014 & 321 & 693 & & 647 & 218 & 429 & \\
\hline
\end{tabular}

transcription factor $(A B R 1)$ were upregulated at $4{ }^{\circ} \mathrm{C}$ but downregulated at $-20^{\circ} \mathrm{C}$. The others were all downregulated at $4{ }^{\circ} \mathrm{C}$ and $-20^{\circ} \mathrm{C}$, including WRKY transcription factors (WRKY11, WRKY17, WRKY24, WRKY41, WRKY48, WRKY53), calcium-binding proteins (CML19, 2 CML27, CML37), ethylene-responsive transcription factors (ERF1A, ERF017, ERF020, ERF109), and dehydration-responsive element-binding protein 1B (DREB1D) (Table 2; Table S11).

\section{Starch and sucrose metabolism pathway}

Starch and sucrose metabolism included two processes: synthesis and decomposition. Six genes with TPM values less than 10 were removed, leaving 4 upregulated and 11 downregulated genes at $4{ }^{\circ} \mathrm{C}$ and 2 upregulated and 13 downregulated genes at $-20^{\circ} \mathrm{C}$ (Table 2). In addition, the leucine-rich repeat receptor-like serine/threonine-protein kinase (LRR$R L K)$ and beta-glucosidase (BGLU12) genes regulating glucose synthesis were significantly upregulated at $4{ }^{\circ} \mathrm{C}$, but the beta-glucosidase (F26G, At4g27290) genes were downregulated. The gene beta-amylase 1 (BAM1), which regulates maltose synthesis, was upregulated, but $B A M 2$ and $B A M 3$ were downregulated. Additionally, a TMV resistance protein was annotated to be upregulated in Prunus mume, indicating a key role of sucrose synthase. The other downregulated genes were galacturonosyl transferase (GATL3, GATL10), cellulose synthase A catalytic subunit 5 (CESA2, CESA6), 1,4-alpha-glucan-branching enzyme 1 (SBE1), isoamylase 1 (ISA1), and APO protein 1 (APO1). Beta-glucosidase (At4g27290) and LRR-RLK were upregulated at $4{ }^{\circ} \mathrm{C}$ but downregulated at $-20^{\circ} \mathrm{C}$ (Table 2; Table S11). 


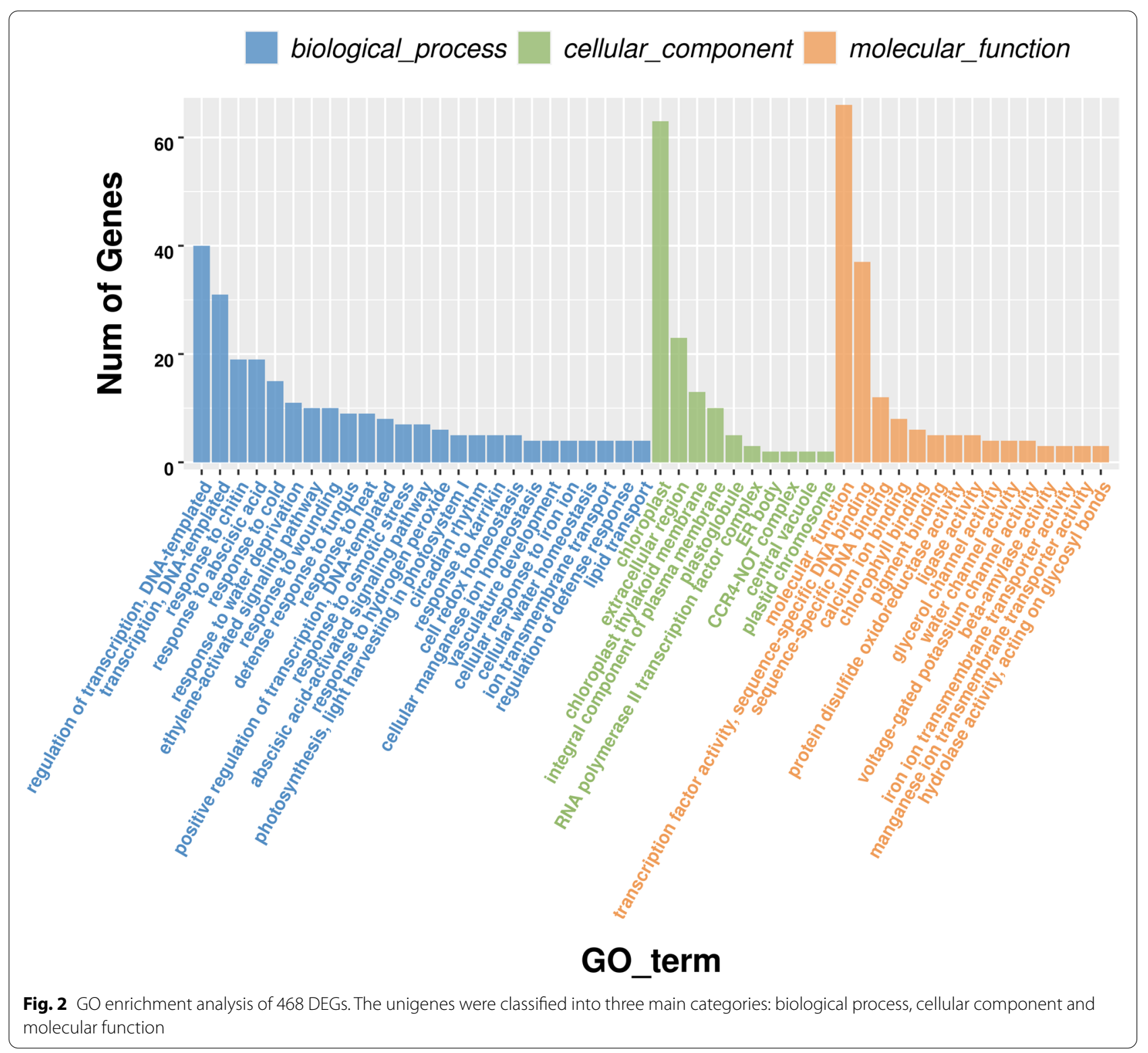

Table 2 Summary of KEGG pathway functional annotations for 468 DEGs

\begin{tabular}{|c|c|c|c|c|c|c|}
\hline \multirow[t]{3}{*}{ Pathway } & \multirow[t]{3}{*}{ ID code } & \multirow{3}{*}{$\begin{array}{l}\text { The number of DEGs in } \\
\text { metabolic pathway }\end{array}$} & \multicolumn{4}{|c|}{ Number of 468 DEGs } \\
\hline & & & \multicolumn{2}{|c|}{$4^{\circ} \mathrm{C}$ vs. $23^{\circ} \mathrm{C}$} & \multicolumn{2}{|c|}{$-20^{\circ} \mathrm{C}$ vs. $23^{\circ} \mathrm{C}$} \\
\hline & & & Up & down & Up & Down \\
\hline Plant-pathogen interaction & ko04626 & 32 & 6 & 26 & 4 & 28 \\
\hline Starch and sucrose metabolism & ko00500 & 21 & 4 & 17 & 3 & 18 \\
\hline Circadian rhythm - plant & ko04712 & 8 & 3 & 5 & 4 & 4 \\
\hline Photosynthesis - antenna proteins & ko00196 & 7 & 7 & 0 & 7 & 0 \\
\hline
\end{tabular}




\section{Circadian rhythm-plant metabolic pathway}

In the circadian rhythm-plant metabolic pathway, 8 genes were significantly different among the three groups, including 3 upregulated and 5 downregulated genes at $4{ }^{\circ} \mathrm{C}$ and 4 upregulated and 4 downregulated genes at $-20^{\circ} \mathrm{C}$ (Table 2). Removing the gene with a TPM value less than 10, among the remaining 7 genes, the APRR 5 and GI 2 genes were upregulated at $4{ }^{\circ} \mathrm{C}$, and $A P R R 5$ was also upregulated at $-20^{\circ} \mathrm{C}$, but its expression was lower at $4{ }^{\circ} \mathrm{C}$. In addition, both MYB23 and $C 1$ were significantly upregulated and annotated as trichome differentiation protein and transcription factor WER, respectively; the other genes were downregulated (Table 2; Table S11).

\section{Photosynthesis - antenna proteins metabolic pathway}

Antenna proteins are the most important part of the light harvesting complex ( $L H C)$ in terms of light energy collection during photosynthesis. In the metabolic pathway of photosynthesis-antenna proteins, 7 genes were significantly upregulated among the three temperature groups. Gene annotation showed that $C A B 2$, a hypothetical protein GLYMA was continuously upregulated under the $4{ }^{\circ} \mathrm{C}$ and $-20^{\circ} \mathrm{C}$ treatments, and its TPM value was 10.64 at $-20^{\circ} \mathrm{C}$. The other six chlorophyll a-b binding protein genes (LHCB3, $2 A B 80,2$ CAB-151, CAB40) showed high expression at $4{ }^{\circ} \mathrm{C}$ but slightly decreased expression at $-20^{\circ} \mathrm{C}$ (Table 2; Table S11).

\section{qRT-PCR validation of the DEGs}

Based on the results of transcriptome annotation under low-temperature stress, eight DEGs related to cold resistance were screened (Fig. 3; Table S12). The fold change in expression was analysed by qRT-PCR before and after low-temperature stress. The results indicated that the expression of the PGR5, CHLH, BBX24, STN7, EXPA8, LRR-RLK and CIPK12 unigenes was upregulated, and that of $b Z I P 60$ was downregulated. The same trend was observed in the high-throughput sequencing analysis. Consequently, these genes mentioned above may be directly correlated with cold resistance in $R$. xanthina f. spontanea, and the results further confirmed the reliability of our transcriptome data.

\section{Discussion}

In view of the lack of genome-wide data for nonmodel plants, high-throughput transcriptome sequencing technology can determine the sequences of each transcriptional fragment and rare transcript for any species. There is no need to understand the genetic information of that species. Thus, this method is crucial to the study nonmodel plants. In the present study, the transcriptional data we obtained were compared with data from known protein databases (Nr, Swiss-Prot, COG, KEGG) according to the principle that if the gene structure is similar, then the function is homologous. The functional genes were annotated with the support of a powerful bioinformatics platform. To date, this platform has been successfully used in previous reports on nonmodel plants [16-20] and provides

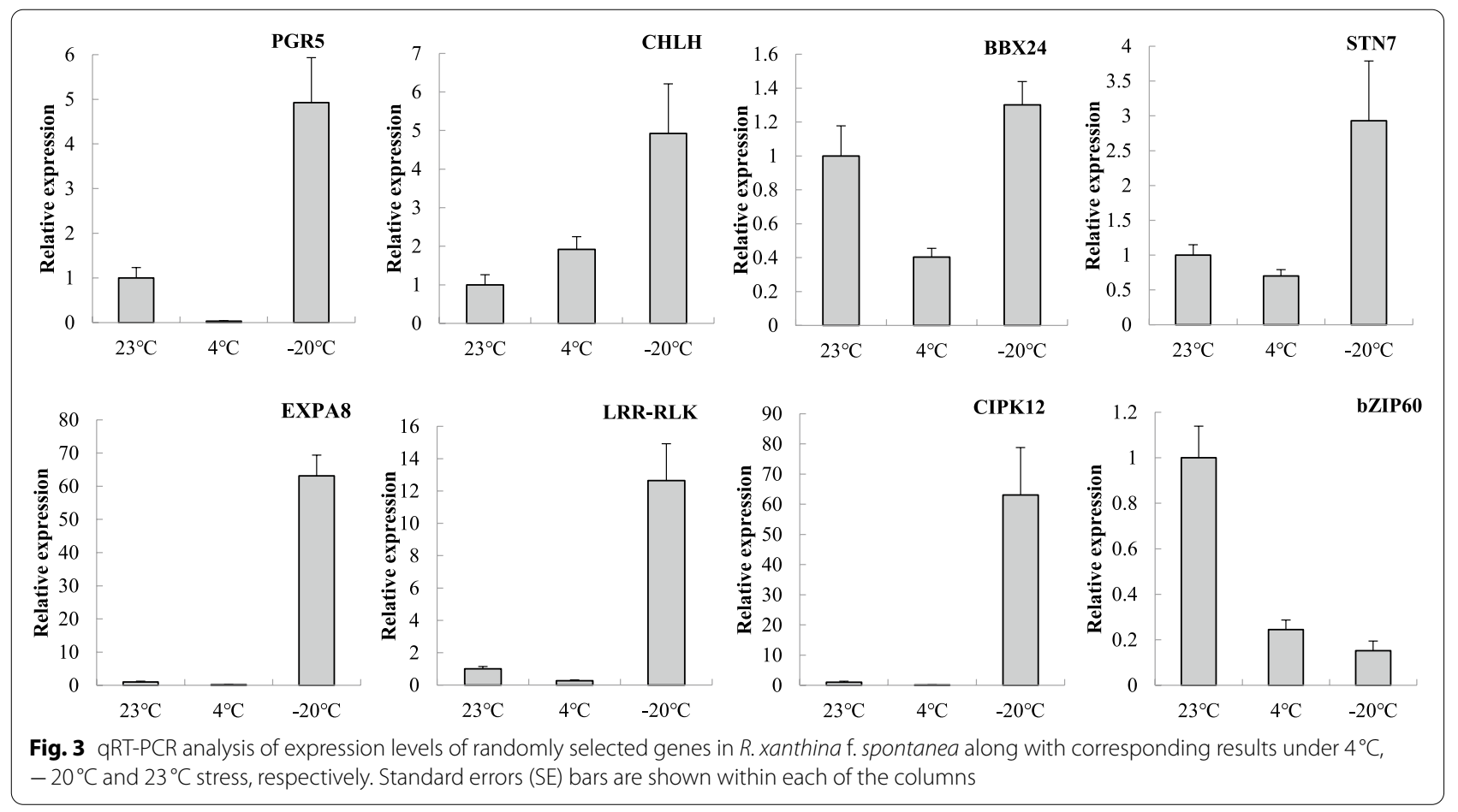


abundant genetic data sources for plant functional genomics. High-throughput sequencing was performed on the leaves of $R$. multiflora under low-temperature stress using an Illumina $\mathrm{HiSeq}^{\mathrm{TM}} 4000$, and a total of 55,084 unigenes were identified. Of these unigenes, 37,303 unigenes (67.7\%) had functional annotations when compared with the Nr database, and the most frequent plant was Fragaria vesca, with $53.5 \%$ of the total unigenes (Fig. S1; Table S3). The results were consistent with those from previous studies [12, 21], where the unigenes represented $32.8 \%$ of $R$. multiflora and $64.6 \%$ of $R$. beggeriana Schrenk genes.

In the present study, there were more downregulated genes than upregulated genes at $4{ }^{\circ} \mathrm{C}$ and $-20^{\circ} \mathrm{C}$ in $R$. xanthina f. spontanea. This result is similar with those of Kou et al. [22] in potato and Zhou et al. [23] in Chinese jujube, which indicated that different cultivars may have similar responses and cold resistance mechanisms. However, these results are not consistent with those of Niu et al. [24] in Prunus persica under freezing stress treatment, in which the number of upregulated genes was greater than that of downregulated genes. At the same time, these results are also different from those of Zhang et al. [12] in R. multiflora, which may be due to the use of different experimental materials. In addition, the results suggested that all the DEGs were more abundant under $44^{\circ} \mathrm{C}$ stress than at $-20^{\circ} \mathrm{C}$ regardless of whether they were upregulated and downregulated, suggesting that related genes may be expressed by cold signals in the early stage of low temperature stress in $R$. xanthina f. spontanea.

By performing KEGG pathway analyses, among all the DEGs of the three treatment groups, the commonly expressed genes were enriched in four pathways: plantpathogen interaction, starch and sucrose metabolism, circadian rhythm-plant and photosynthesis-antenna proteins (Table 2). In $R$. beggeriana, many DEGs were enriched in starch and sucrose metabolism and the plantpathogen interaction pathway at $4{ }^{\circ} \mathrm{C}$ [21]. In addition, $\mathrm{Du}$ et al. [25] performed transcriptome analysis under cold stress in Brassica napus L., and the results indicated that many DEGs were enriched in the circadian rhythm-plant, plant-pathogen interaction metabolic, plant hormone signal transduction and secondary metabolic pathways. Photosynthesis was the first metabolic process inhibited during chilling injury [26]. Chloroplasts are the main organelles affected by cold conditions [2].

In the present study, 7 light-harvesting complex II chlorophyll $\mathrm{a} / \mathrm{b}$ binding protein genes enriched in the photosynthesis-antenna protein pathway were significantly upregulated, and the gene expression increased dramatically, especially in $C A B-151$ and $C A B 40$. Therefore, the genes encoding light-harvesting complex II chlorophyll $\mathrm{a} / \mathrm{b}$ binding proteins were upregulated under cold stress in $R$. xanthina f. spontanea, improving photosynthesis and enhancing cold resistance. The final products of photosynthesis were starch and sucrose. Based on the starch and sucrose metabolism pathway, we found that many genes were upregulated or downregulated and were involved in complex metabolic reactions to adapt to low temperature stress. Of these genes, only the $L R R-R L K$ gene of the leucine-rich repeat receptorlike serine/threonine-protein kinase family was upregulated at $4{ }^{\circ} \mathrm{C}$ and $-20^{\circ} \mathrm{C}$, indicating that the $L R R-R L K$ gene may play an important role in regulating cold stress responses. Additionally, there was a circadian rhythm to the expression of $c a b$, which may be controlled by the biological clock [27-29]; this was consistent with the pathways enriched in circadian rhythm plants. The timing function of the plant biological clock is related to the level of carbohydrates in plant cells, and carbohydrates are produced by photosynthesis in plants, which is a key metabolic input of the plant biological clock. Carbohydrates, as feedback substances that accumulate in plants, can regulate the timing and reset function of the plant biological clock to the external cycle [30]. Regarding GO enrichment analyses of the DEGs common to the three treatment groups, for cellular components, most DEGs were enriched in chloroplasts, extracellular regions, and chloroplast thylakoid membranes, and the results were consistent with the location of photosynthesis. In comparison to those at $4^{\circ} \mathrm{C}$, more DEGs were enriched in secondary metabolic pathways at $-20^{\circ} \mathrm{C}$, suggesting that cold signalling enhanced antenna protein genes during cold stress. Furthermore, to enhance photosynthesis, promote carbon metabolism, and strengthen cold resistance in $R$. xanthina f. spontanea, with a decrease in temperature, a number of genes that regulate metabolism were activated to protect against low temperature. In this study, the response mechanism under cold stress was systematically analysed at the transcriptional level in $R$. xanthina f. spontanea to elucidate cold-related metabolic pathways and lay a foundation for exploring the key functional genes associated with cold tolerance. Therefore, it is of great significance to accelerate the progress of genetic improvement of cold tolerance in rose.

\section{Conclusions}

Our study is the first to report on the response to cold stress at the transcriptome level in $R$. xanthina f. spontanea, which can provide a theoretical basis for further studies on the molecular mechanism of cold resistance in rose. Important genes involved in plant-pathogen interactions, starch and sucrose metabolism, circadian rhythm-plant and photosynthesis-antenna proteins were significantly enriched under low-temperature stress, which most of the genes were downregulated. In comparison to $4{ }^{\circ} \mathrm{C}$, secondary metabolites play an important 
role at $-20^{\circ} \mathrm{C}$. The results of this study may be beneficial for further studies on cold tolerance mechanisms in rose and other plants.

\section{Methods}

\section{Plant materials and low-temperature treatment}

The plant materials of $R$. xanthina $\mathrm{f}$. spontanea used in our study were planted in Liaoning Research Institute of Cash Crops, Liaoning Academy of Agricultural Sciences, Liaoning, 110,161, China. It is necessary to obtaining permissions to collect these samples, Jiajun Lei professor (College of Horticulture, Shenyang Agricultural University, Liaoning 110,866, China) undertook the formal identification of the samples, and its detailed information was described based on Help Me Find (https://www.helpmefind.com/ gardening $/$ l.php? $=2.46960 .1)$. Cutting propagation was performed in the greenhouse; annual cutting seedlings with the same growth vigour and management conditions were placed in a variable temperature $\left(23^{\circ} \mathrm{C}\right)$ climate chest 1 week prior (16h light, $8 \mathrm{~h}$ darkness and $70 \%$ relative humidity), and the temperature was dropped $2^{\circ} \mathrm{C}$ per hour to $4{ }^{\circ} \mathrm{C}$ and $-20^{\circ} \mathrm{C}$. This temperature was maintained for $12 \mathrm{~h}$ and then heated to $23^{\circ} \mathrm{C}$ at $2{ }^{\circ} \mathrm{C}$ per hour. The leaf blades were quickly placed in liquid nitrogen and stored at $-80^{\circ} \mathrm{C}$. All the experiments were repeated three times.

\section{RNA extraction, library construction and sequencing} Total RNA was extracted using TRIzol reagent (Invitrogen, CA, USA) following the manufacturer's procedure. The total RNA quantity and purity were analysed by a Bioanalyzer 2100 and RNA 6000 Nano LabChip Kit (Agilent, CA, USA) with a RIN number $>7.0$. Approximately $10 \mu \mathrm{g}$ of total RNA representing a specific adipose type was subjected to isolate poly(A) mRNA purification with poly-T oligo-attached magnetic beads (Invitrogen, CA, USA). Following purification, the poly(A) or poly $(\mathrm{A})+\mathrm{RNA}$ fraction was fragmented into small pieces using divalent cations under elevated temperature. Then, the cleaved RNA fragments were reverse-transcribed to create the final cDNA library in accordance with the protocol for the mRNA-Seq sample preparation kit (Illumina, San Diego, USA), and the average insert size for the paired-end libraries was $300 \mathrm{bp}$ ( $\pm 50 \mathrm{bp})$. Then, paired-end sequencing was performed by an Illumina HiSeq 4000 (LC-Bio, China) following the vendor's recommended protocol. The raw sequence data have been submitted to the NCBI Short Read Archive with accession code PRJNA724822.

\section{De novo assembly, unigene annotation and differential expression analysis}

First, de novo assembly, functional annotation and classification of the unigenes were performed, and cutadapt [31] and in-house Perl scripts were used to remove the reads that contained adaptor contamination, low-quality bases and undetermined bases. And, the reliability of the unigenes assembly was tested using BUSCO (ver. 5.1.2) (Fig. S4). Then, sequence quality was verified using FastQC (http://www.Bioinformatics.babraham.ac.uk/projects/ fastqc/), including the Q20, Q30 and GC content of the clean data. All downstream analyses were based on clean data with high quality. De novo assembly of the transcriptome was performed with Trinity 2.4.0 [32]. Trinity groups transcripts into clusters based on shared sequence content.

All assembled unigenes were aligned against the NCBI nonredundant protein sequences (Nr) (http://www.ncbi. nlm.nih.gov/), GO (http://www.geneontology.org), SwissProt (http://www.expasy.ch/sprot/), KEGG (http://www.genome. jp/kegg/) and eggnog (http://eggnogdb.embl.de/) databases using DIAMOND [33] with a threshold of $E$ value $<0.00001$.

Differentially expressed unigene analysis based on Salmon [34] was used to determine the expression level for unigenes by calculating transcripts per million (TPM) [35]. The DEGs were selected with $\log 2$ (fold change) $>1$ or $\log 2$ (fold change $)<-1$ and with statistical significance $(p<0.05)$ by

Table 3 The primers used for qRT-PCR in this study

\begin{tabular}{|c|c|c|c|c|}
\hline No. & Gene ID & Code & Forward primer (F) & Reverse primer (R) \\
\hline 1 & TRINITY_DN25157_C0_g1 & PGR5 & AGGGCACAACCCATGATGAA & TTCGGCTCTTAGACAAAGGCAA \\
\hline 2 & TRINITY_DN31568_c1_g5 & $\mathrm{CHLH}$ & AGATGAGCCAGTTGAACAGAA & AGTAGGAGCCTGAAGCATTG \\
\hline 3 & TRINITY_DN27059_c1_g3 & $\mathrm{BB} \times 24$ & CAATAGCCTCTCTGCCAACCA & GGTGGCTCTACGCTACTTGTT \\
\hline 4 & TRINITY_DN30314_c0_g1 & STN7 & CCAGATGGGTTACTCGGCTAA & GACTTCTTCTTAGGCTTCGTTTCC \\
\hline 5 & TRINITY_DN30720_c0_g10 & EXPA8 & GGGTCTTGTTACGAAATGCGATGT & CAGAAGTTGGTGGCGGTGAC \\
\hline 6 & TRINITY_DN32852_c1_g1 & LRR-RLK & GGACCGAGACCTCAATGCTAAGA & TCCTGGTGCTAATGTGAGTGTTCT \\
\hline 7 & TRINITY_DN29285_c0_g3 & CIPK12 & CGAAGAACAACCCGCTCCTCCTC & CGCCCTCGTCGGTCTTGATGT \\
\hline 8 & TRINITY_DN31926_c0_g6 & bZIP60 & TCTTCGTCGTCGTCGTCATC & TTCATCAGCATGTCCTCAACCT \\
\hline 9 & Actin & & CCTCTATGCCAGTGGTCGTACAA & GCCAGGTCAAGTCGCAGAATG \\
\hline 10 & $18 s$ & & CAACCATAAACGATGCCGA & AGCCTTGCGACCATACTCC \\
\hline
\end{tabular}


the R package edgeR [36]. Next, GO and KEGG enrichment analyses were repeated based on the DEGs identified by the OmicStudio tools at https://www.omicstudio.cn/tool/22.

\section{qRT-PCR validation}

The leaves of $R$. xanthina $\mathrm{f}$. spontanea were collected and treated at $23^{\circ} \mathrm{C}, 4^{\circ} \mathrm{C}$ and $-20^{\circ} \mathrm{C}$, and $10 \mathrm{DEGs}$ selected at random were used for qRT-PCR validation. Total RNA was extracted by using a polysaccharide polyphenol Plant RNA Isolation Kit (N1005, Biobase Technologies Co., Ltd., ChengDu, China), and reverse transcription of cDNA was performed using a TUREscript 1st Stand cDNA Synthesis Kit (Aidlab Biotechnologies Co., Ltd., Beijing, China). Primers were designed using Beacon designer 7.9 software for qRT-PCR, and primers are listed in Table 3. qRTPCR assays were performed on an Analytik Jena-qTOWER 2.2 (Germany) with $2 \times$ SYBR $^{\circledR}$ Green SuperMix (DF, China) and amplified with $1 \mu \mathrm{L}$ of cDNA template, $5 \mu \mathrm{L}$ of $2 \times$ SYBR Green Super Mix, and $0.5 \mu \mathrm{L}$ of each primer to a final volume of $10 \mu \mathrm{L}$ with water. The amplification programme consisted of one cycle at $95^{\circ} \mathrm{C}$ for $3 \mathrm{~min}$, followed by 59 cycles of $95^{\circ} \mathrm{C}$ for $30 \mathrm{~s}$ and $60^{\circ} \mathrm{C}$ for $30 \mathrm{~s}$. The relative expression levels were calculated by the $2^{-\triangle \triangle \mathrm{CT}} \operatorname{method}[37]$.

\section{Abbreviations}

DEGs: Differentially Expressed Genes; KEGG: Kyoto Encyclopedia of Genes and Genomes; qRT-PCR: Quantitative Real-time PCR; GO: Gene Ontology.

\section{Supplementary Information}

The online version contains supplementary material available at https://doi. org/10.1186/s12870-021-03246-5.

Additional file 1: Table S1. Overview of the sequencing and assembly of the transcriptome.

Additional file 2: Table S2. N50 of transcripts or unigenes from nine samples.

Additional file 3: Table S3. Species distribution frequency of the BLAST hits based on $\mathrm{Nr}$ database.

Additional file 4: Table S4. GO classification of the assembled unigenes in R. xanthina f. spontanea. (XLSX $7387 \mathrm{~kb}$ )

Additional file 5: Table S5. KEGG function classification the assembled unigenes in R. xanthina f. spontanea. (XLSX2156 kb)

Additional file 6: Table S6. $G O$ enrichment analysis of DEGs at $4^{\circ} \mathrm{C}$ vs. $23^{\circ} \mathrm{C}$ in R. xanthina f. spontanea. (XLSX212 kb)

Additional file 7: Table S7. GO enrichment analysis of DEGs at $-20^{\circ} \mathrm{C}$ vs. $23^{\circ} \mathrm{C}$ in R.xanthina f. spontanea. (XLSX $117 \mathrm{~kb}$ )

Additional file 8: Table S8. KEGG enrichment analysis of DEGs at $4^{\circ} \mathrm{C}$ vs. $23^{\circ} \mathrm{C}$ in R. xanthina f. spontanea. (XLSX $743 \mathrm{~kb}$ )

Additional file 9: Table S9. $\mathrm{KEGG}$ enrichment analysis of DEGs at $-20^{\circ} \mathrm{C}$ vs. $23^{\circ} \mathrm{C}$ in R.xanthina f. spontanea. (XLSX $318 \mathrm{~kb}$ )

Additional file 10: Table S10. GO enrichment analysis of 468 DEGs under low-temperature stress in R. xanthina f. spontanea. (XLSX 91 kb)

Additional file 11: Table S11. KEGG enrichment analysis of 468 DEGs under low-temperature stress in R. xanthina f. spontanea. (XLSX66 kb)

Additional file 12: Table S12. qRT-PCR analysis of expression levels under low-temperature stress in R. xanthina f. spontanea. (XLSX 16 kb)
Additional file 13: Figure S1. Species distribution of the BLAST hits for each unigenes based on $\mathrm{Nr}$ database.

Additional file 14: Figure S2. GO enrichment analysis of the assembled unigenes. (DOCX $63 \mathrm{~kb}$ )

Additional file 15: Figure S3. KEGG pathway functional category of the assembled unigenes. (DOCX $99 \mathrm{~kb}$ )

Additional file 16: Figure S4. The BUSCO assessment results.

\section{Acknowledgements}

Not applicable.

\section{Authors' contributions}

$J J L$ and XFY conceived and designed research; DFZ conducted the experiments; LX collected and analyzed the data; DFZ wrote the manuscript; CM and ZL provided valuable suggestions on the manuscript; CW and XFY revised the manuscript. All the authors read and approved the manuscript.

\section{Funding}

This work was financially supported by Doctoral Research Fund Program of Inner Mongolia Minzu University (grant no. BS561). The finding body provided only the expenses and fees of this study. The design of the study and collection, analysis, and interpretation of data and in writing the manuscript were performed by the contributing authors.

\section{Availability of data and materials}

All data generated in this study are included with its supplementary material. The datasets during the study are deposited in NCBI Sequence Read Archive (SRA) (http://www.ncbi.nlm.nih. gov/ sra /), the accession number is: PRJNA724822.

\section{Declarations}

\section{Ethics approval and consent to participate}

Plant materials (Rosa xanthina f. spontanea.) used in this study were collected from our experimental base (Liaoning Research Institute of Cash Crops, Liaoyang, Liaoning, 110161, China). All plant materials used in this experiment were not endangered materials or species. Study complies with relevant institutional, national, and international guidelines and legislation.

\section{Consent for publication}

Not applicable.

\section{Competing interests}

The authors declare that they have no competing interests.

\section{Author details}

${ }^{1}$ Liaoning Academy of Agricultural Sciences, Shenyang 110161, Liaoning, China. ${ }^{2}$ Agricultural College, Inner Mongolia Minzu University, Tongliao 028000, China. ${ }^{3}$ College of Horticulture, Shenyang Agricultural University, Shenyang 110866, Liaoning, China. ${ }^{4}$ College of Life Sciences and Food Engineering, Inner Mongolia Minzu University, Tongliao 028000, China.

Received: 2 February 2021 Accepted: 29 September 2021

Published online: 15 October 2021

\section{References}

1. Lata C, Prasad M. Role of DREBs in regulation of abiotic stress responses in plants. J Exp Bot. 2011;62:4731-48.

2. Theocharis A, Clement C, Barka EA. Physiological and molecular changes in plants grown at low temperatures. Planta. 2012;235:1091-105.

3. Wang Z, Gerstein M, Snyder M. RNA-Seq: a revolutionary tool for transcriptomics. Nat Rev Genet. 2009;10:57-63.

4. Samuel PH, Wu YJ, Joel AK. Gene expression profiling of plant responses to abiotic stress. Functional \& Integrative Genomics. 2003;3(3):105-11. 
5. Wang R, Wang X, Liu K, Zhang XJ, Zhang LY, Fan SJ. Comparative transcriptome analysis of halophyte Zoysia macrostachya in response to salinity stress. Plants. 2020;9:458-75.

6. Wang XC, Zhao QY, Ma CL, Zhang ZH, Cao HL, Kong YM, et al. Global transcriptome profiles of Camellia sinensis during cold acclimation. BMC Genomics. 2013;14:415-28.

7. Chen JH, Tian QQ, Pang T, Jiang LB, Wu RL, Xia XL, et al. Deep- sequencing transcriptome analysis of low temperature perception in a desert tree, Populus euphratica. BMC Gen. 2014;15:326-40.

8. Han Y, Wan HH, Cheng TR, Wang J, Yang WR, Pan HT, et al. Comparative RNA-seq analysis of transcriptome dynamics during petal development in Rosa chinensis. Sci Rep. 2017;7:43382.

9. Huang XL, Yan HQ, Zhai LS, Yang ZT, Yi Y. Characterization of the Rosa roxbunghii tratt transcriptome and analysis of MYB genes. PLoS One. 2019;14:e0203014.

10. Qi WC, Chen X, Fang PH, Shi SC, Li JJ, Liu XT, et al. Genomic and transcriptomic sequencing of Rosa hybrida provides microsatellite markers for breeding, flower trait improvement and taxonomy studies. BMC Plant Biol. 2018;18:1-12.

11. Yan X, Zhang X, Lu M, He Y, An H. De novo sequencing analysis of the Rosa roxburghii fruit transcriptome reveals putative ascorbate biosynthetic genes and EST-SSR markers. Gene. 2015;561:54-62.

12. Zhang XY, Zhang JZ, Zhang WW, Yang T, Xiong Y, Che DD. Transcriptome sequencing and de novo analysis of Rosa multiflora under cold stress. Acta Physiol Plant. 2016;38:164-76.

13. Zhang F, Ji SJ, Wei BD, Cheng SC, Wang YJ, Hao J, et al. Transcriptome analysis of postharvest blueberries (Vaccinium corymbosum 'Duke') in response to cold stress. BMC Plant Bio. 2020;20(2):493-501.

14. Michele VR, Laura VR, Ahmed S, Lucimara K, Steven JC, Bernanda C, et al. Genome-wide expression of low temperature response genes in Rosa hybrida L. Plant Physiol Biochem. 2020;146:238-48.

15. Ma Y, Chen JY. A study to evaluate cold hardiness of some parent plants and hybrid seedlings during breeding new cultivars for establishing a rejuvenation rose group. J Beijing Forestry University. 1992;1:60-5 (In Chinese with English abstract).

16. Arslan M, Devisetty UK, Porsch M, Grosse I, Michalski SG. RNA-Seq analysis of soft rush (Juncus effusus): transcriptome sequencing, de novo assembly, annotation, and polymorphism identification. BMC Gen. 2019;20:489-500.

17. Du F, Wu Y, Zhang L, Li X, Zhao X, Wang W, et al. De novo assembled transcriptome analysis and SSR markers development of a mixture of six tissues from Lilium oriental hybrid 'Sorbonne.' Plant Mol Biol Rep. 2015;33:281-93.

18. Hyun TK, Lee S, Rim Y, Kumar R, Han X, Lee SY, et al. De-novo RNA sequencing and metabolite profiling to identify genes involved in anthocyanin biosynthesis in Korean black raspberry (Rubus coreanus Miquel). PLoS One. 2014;9:e88292.

19. Lateef A, Prabhudas SK, Natarajan P. RNA sequencing and de novo assembly of Solanum trilobatum leaf transcriptome to identify putative transcripts for major metabolic pathways. Sci Rep. 2018;8:76-80.

20. Younesi-Melerdi E, Nematzadeh GA, Pakdin-Parizi A, Bakhtiarizadeh MR, Motahari SA. De novo RNA sequencing analysis of Aeluropus littoralis halophyte plant under salinity stress. Sci Rep. 2020;10:9148.

21. Liu Q. Physiological mechanisms and transcriptome analysis on cold resistance of Rosa beggeriana Schrenk. Master's thesis. Beijing: Chinese Academy of Agricultural Sciences; 2017. (In Chinese with English abstract)

22. Kou S, Chen L, Tu W, Federico S, Wang YM, Liu J, et al. The arginine decarboxylase gene $A D C 1$, associated to the putrescine pathway, plays an important role in potato cold-acclimated freezing tolerance as revealed by transcriptome and metabolome analyses. Plant J. 2018;96:1283-98.

23. Zhou HY, He Y, Zhu YS, Li MY, Song S, Bo WH, et al. Comparative transcriptome profiling reveals cold stress responsiveness in two contrasting Chinese jujube cultivars. BMC Plant Biol. 2020;20:240.

24. Niu RX, Zhao XM, Wang CB, Wang FL. Transcriptome profiling of Prunus persica branches reveals candidate genes potentially involved in freezing tolerance. Sci Hortic. 2019;259:108775.

25. Du CF, Hu KN, Xian SS, Lin CQ, Fan JC, Tu TX, et al. Dynamic transcriptome analysis reveals AP2/ERF transcription factors responsible for cold stress in rapeseed (Brassica napus L.). Mol Gen Genomics. 2016;291(3):1053-67.

26. Hu WH, Wu Y, Zeng JZ, He L, Zeng QM. Chill-induced inhibition of photosynthesis was alleviated by 24-epibrassinolide pretreatment in cucumber during chilling and subsequent recovery. Photosynthetica. 2010;48(4):537-44.
27. Millar AJ, Kay SA. Circadian control of cab gene transcription and mRNA accumulation in Arabidopsis. Plant Cell. 1991;3(5):541.

28. Legocka J, Schneider J, Jackowski G. Cytokinin controlled expression of the apoprotein of the light-harvesting complex of photosystem II in the tissue culture of Dianthus caryophyllus new nam. I. protein identification. Acta Physiologiae Plantarum. 1990;12(4):303.

29. Riesselmann S, Piechulla B. Diurnal and circadian light harvesting complex and quinone B-binding protein synthesis in leaves of tomato (Lycopersicum esculentum). Plant physiol. 1992;11(2):219.

30. Haydon Michael J, Olga M, Robertson Fiona C, Hubbard Katharine E, Webb Alex AR. Photosynthetic entrainment of the Arabidopsis thaliana circadian clock. Nature. 2013;502:7473.

31. Martin M. Cutadapt removes adapter sequences from high-throughput sequencing reads. Embnet Journal. 2011;17:10-2.

32. Grabherr MG, Haas BG, Yassour M, Levinet JZ, Thompson DA. Full-length transcriptome assembly from RNA-seq data without a reference genome. Nature Bio. 2011;29:644-52.

33. Buchfink B, Xie C, Huson DH. Fast and sensitive protein alignment using diamond. Nat Methods. 2015;12:59-60.

34. Patro R, Duggal G, Love MI, Irizarry RA, Kingsford C. Salmon provides fast and bias-aware quantification of transcript expression. Nat Methods. 2017;14:417-9.

35. Mortazavi A, Williams BA, Mc CK, Schaeffer L, Word B. Mapping and quantifying mammalian transcriptomes by RNA-seq. Nat Methods. 2008;5:621-8.

36. Robinson MD, McCarthy DJ, Smyth GK. EdgeR: a bioconductor package for differential expression analysis of digital gene expression data. Bioinformatics. 2010;26:139-40.

37. Livak KJ, Schmittgen TD. Analysis of relative gene expression data using real-time quantitative $P C R$ and the $2^{-\triangle \triangle C T}$ method. Methods. 2001;25:402-8.

\section{Publisher's Note}

Springer Nature remains neutral with regard to jurisdictional claims in published maps and institutional affiliations.

Ready to submit your research? Choose BMC and benefit from

- fast, convenient online submission

- thorough peer review by experienced researchers in your field

- rapid publication on acceptance

- support for research data, including large and complex data types

- gold Open Access which fosters wider collaboration and increased citations

- maximum visibility for your research: over 100M website views per year

At BMC, research is always in progress.

Learn more biomedcentral.com/submissions 